TERMINAL, 117 (POST PRINT)

DOI : HTTPS://DOI.ORG/10.4000/TERMINAL. 1130

\title{
Les objets numériques : perception des utilisateurs et tactiques d'appropriation
}

\author{
Samuel GUILLEMOT, Univ Brest, LEGO, Quimper, France \\ Andréa GOURMELEN, Univ Montpelier, MRM, Montpelier, France \\ Klara KOVESI, CNAM Paris, CRF, France \\ Annick TAMARO, Univ Brest, LEGO, Quimper, France
}

Cet article est issu d'un projet de recherche M@rsouin, qui a bénéficié d'un financement de la région Bretagne. Les auteurs remercient également les deux évaluateurs pour leurs conseils qui ont permis d'améliorer sensiblement cet article.

TO CITE: GUILLEMOT S. ; GOURMELEN A., KOVESI K., AND TAMARO, A. (2015), LES OBJETS NUMERIQUES : PERCEPTION DES UTILISATEURS ET TACTIQUES D'APPROPRIATION, REVUE TERMINAL, 117, HTTPS://JOURNALS.OPENEDITION.ORG/TERMINAL/1130

Une partie des objets autrefois tangibles, tels que les photos, les correspondances, les films et les musiques est aujourd'hui démocratisée sous forme numérique. Peut-on pour autant parler d'objets numériques ? Est-il possible de se les approprier ? Si oui, par quel(s) processus ? Cet article prend le parti d'aborder le numérique en termes d'objets, supports de diverses formes d'appropriation et de manipulations. Ces questions ne sont pas dénuées d'intérêt ; en témoigne le cas du célèbre acteur américain Bruce Willis qui a menacé de poursuivre en justice la société Apple quand il s'est rendu compte qu'il ne pouvait pas transmettre sa bibliothèque iTunes à ses enfants (Sears, 2012).

Dans un premier temps, nous allons tâcher de répondre à ces questions d'un point de vue théorique à travers une revue de la littérature centrée autour des concepts d'appropriation légale et sentimentale (partie 1). Ensuite, nous mettrons en évidence le point de vue des utilisateurs grâce à une étude qualitative conduite auprès de 24 personnes d'horizons variés en termes de genre, d'âge et d'expertise (partie 2). Ces différentes étapes permettront alors d'identifier trois configurations en matière d'appropriation des objets numériques, chacune avec ses propres spécificités : les objets numériques fonctionnels, miroirs et totems (partie 3). Dès lors, cette recherche apporte alors un regard complémentaire aux recherches sur les identités et traces numériques (Coutant et Stenger, 2013), au sens où elle s'intéresse à ce que l'individu possède et pas uniquement à ce qu'il est dans le monde virtuel.

\section{APPROPRIATION DES OBJETS NUMERIQUES : DISTINCTIONS THEORIQUES}

Dans cette première section, nous allons poser les fondements théoriques de notre recherche en définissant l'objet numérique (1-1). Nous nous interrogerons également sur des considérations juridiques et affectives - deux modes d'appropriation des objets - dans le but de différencier les propriétés numériques (1-2) des possessions numériques (1-3).

\section{1-1. De l'objet à l'objet numérique}


A notre connaissance aucune recherche scientifique ne traite des objets numériques en tant que support d'appropriation. C'est pourquoi nous proposons notre propre définition en nous basant sur le dictionnaire Larousse qui définit un objet comme « toute chose concrète, perceptible par la vue, le toucher ». Dans ce sens, un objet numérique peut être composé de trois dimensions

-des données : l'ADN de l'objet numérique, il s'agit d'un contenu composé d'une suite de zéros et de uns ;

-une interface permettant de leur donner du sens (par exemple un logiciel permettant de lire et organiser les données);

-un terminal contribuant à tangibiliser et à «prendre en main » les deux dimensions précédentes (ex : ordinateur, smartphone, baladeur mp3/mp4).

Les recherches actuelles se focalisent sur l'objet numérique sans vraiment distinguer ces trois dimensions. Certaines se concentrent sur la question de la matérialité. Par exemple, Magaudda (2012) montre que l'avènement du digital a conduit à la suppression d'objets physiques (CD, DVD). Dans le même temps, cela a entrainé le développement d'autres objets physiques permettant de les lire (baladeur Mp3, smartphone, tablette, etc.) ou de les stocker (carte mémoire, disque dur, clé USB). D'autres recherches se focalisent sur l'adoption des objets numériques lorsqu'il existe une contrepartie physique, sous forme de photos, de musiques, de correspondances, etc. Dans la majorité des cas, pour un même objet, les deux formats (numérique et physique) coexistent. Ainsi, une récente étude menée sur un échantillon représentatif de foyers américains (Venkatesh et Dunkle, 2012) montre que si un quart des individus a adopté exclusivement le numérique pour les photos, un autre quart rejette ce format, tandis que les $50 \%$ restants adoptent les deux formats dans des proportions diverses. Dans la même logique, $61 \%$ des foyers ont remplacé de manière totale ou partielle leurs archives de documents personnels (dossiers médicaux, factures, dossiers d'assurance, documents d'imposition, etc.). Finalement, il semble que les individus procèdent par arbitrage entre les avantages des supports physiques et ceux des formats numériques: une immense possibilité de stockage, une personnalisation possible contre des phénomènes sensoriels comme le toucher, les odeurs, etc. (Kirk et Sellen, 2010 ; Llamas et Belk, 2012).

\section{1-2. L'appropriation légale et la propriété numérique}

L'appropriation est l'action de faire sien, de s'attribuer la propriété de quelque chose (Le Petit Robert). Un premier moyen d'appropriation des objets numériques est l'appropriation légale. Toujours selon le dictionnaire Le Petit Robert, la propriété est définie comme le « droit d'user, de jouir et de disposer d'une chose d'une manière exclusive et absolue sous les restrictions établies par la loi ». Dans ce cadre, si la question de la propriété de la partie tangible de l'objet numérique (le terminal) ne pose pas de problème ; les règles de propriété des aspects intangibles de l'objet sont, en revanche, plus complexes. A noter que l'on écarte ici le cas où l'objet numérique est considéré comme une auvre de l'esprit, ce qui ouvre dans le droit français à droit d'auteur'

\footnotetext{
${ }^{1}$ Code de la propriété intellectuelle, article L111-1 : «L'auteur d'une œuvre de l'esprit jouit sur cette œuvre, du seul fait de sa création, d'un droit de propriété incorporelle exclusif et opposable à tous. »
} 
Les contours mouvants de la propriété des données. La propriété des données numériques plus ou moins personnelles, produites ou non par l'utilisateur - est évolutive et très contextuelle. D'une part, ces données peuvent faire l'objet d'une appropriation « légale » par des prestataires de service sur la base contractuelle de conditions d'utilisation. Par exemple, en stockant vos photos numériques sur la plateforme Dropbox, les conditions d'utilisation stipulent qu' : «en utilisant nos services, vous nous fournissez vos fichiers, contenus, e-mails, contacts [...] Vos Données vous appartiennent. À l'exception de droits limités nous permettant d'offrir les Services $»^{2}$. D'autre part, elles peuvent faire l'objet d'une appropriation souvent «illégale » par les internautes sur la base d'affinités symboliques ou pratiques. En effet, les individus se meuvent sur le réseau informatique mondial et dans le cybermarché comme s'ils étaient dans un espace public en échangeant, partageant, piochant des musiques, vidéos, images et informations (Bardhi et Eckhardt, 2002).

Le droit d'usage pour les interfaces. De plus en plus de prestataires de services tels que Deezer ou i-Tunes utilisent la notion de droit d'usage et le limite au droit d'accès. Ce droit permet d'utiliser l'interface pour accéder au contenu mais ce dernier reste personnel (car attaché à une personne). Il est donc non cessible et non transmissible. Aussi, le contenu (ici, les fichiers musicaux) ne nous appartient pas légalement; il s'avère donc impossible d'en transférer la propriété à autrui (Bellamy et al, 2014).

\section{1-3. L'appropriation sentimentale et la possession numérique}

Certains objets ont plus d'importance que d'autres, en raison notamment de leur rôle ou de leur statut (porteurs de souvenirs). Dans ce cas, ces objets sont valorisés indépendamment de leur valeur monétaire (Kleine et al, 1995). C'est dans ce sens d'attachement affectif (appropriation sentimentale de l'objet) que nous entendrons donc le terme de «possession ». Ce terme fait partie intégrante de la théorie de l'extended self de Belk (1988, 2013), qui met en avant l'existence d'un soi central (core self) autour duquel d'autres éléments caractéristiques de soi gravitent à la manière des électrons de l'atome (il s'agit alors de l' «extended self», ou du soi étendu). Un individu peut ainsi inclure dans son «soi étendu» des objets, personnes, lieux, idées, expériences, qui s'inscrivent dans son histoire personnelle. Dans ce cadre, le terminal (smartphone, ordinateur, etc) peut atteindre ce statut, les données numériques également en raison de leur caractère personnalisable ou encore des efforts effectués pour les sauvegarder (Denegri-Knott, Watkins et Wood, 2012). Cependant, l'attachement ne serait pas aussi fort que pour un bien tangible (Belk, 2013). En effet, les données numériques (e-mails, e-cards, e-books, journaux, fichiers audio/vidéo, etc.) sont perçues comme moins «authentiques » car facilement effaçables et remplaçables (Siddiqui et Turley, 2006). Néanmoins, Cushing (2012) note des différences générationnelles : les plus jeunes s'accapareraient plus facilement les objets numériques que les plus de 60 ans.

\footnotetext{
${ }^{2}$ https://www.dropbox.com/privacy\#terms
} 
En synthèse, les objets numériques se composent de 3 dimensions: des données, des interfaces et des terminaux. Nous avons identifié deux types d'appropriation de ces objets : en lien avec la propriété juridique et/ou en lien avec la proximité affective. Ces trois notions sont imbriquées : un individu peut être attaché à des objets qui ne lui appartiennent pas et inversement (figure 1)

Figure 1 : Dimensions et types d'appropriation des objets numériques
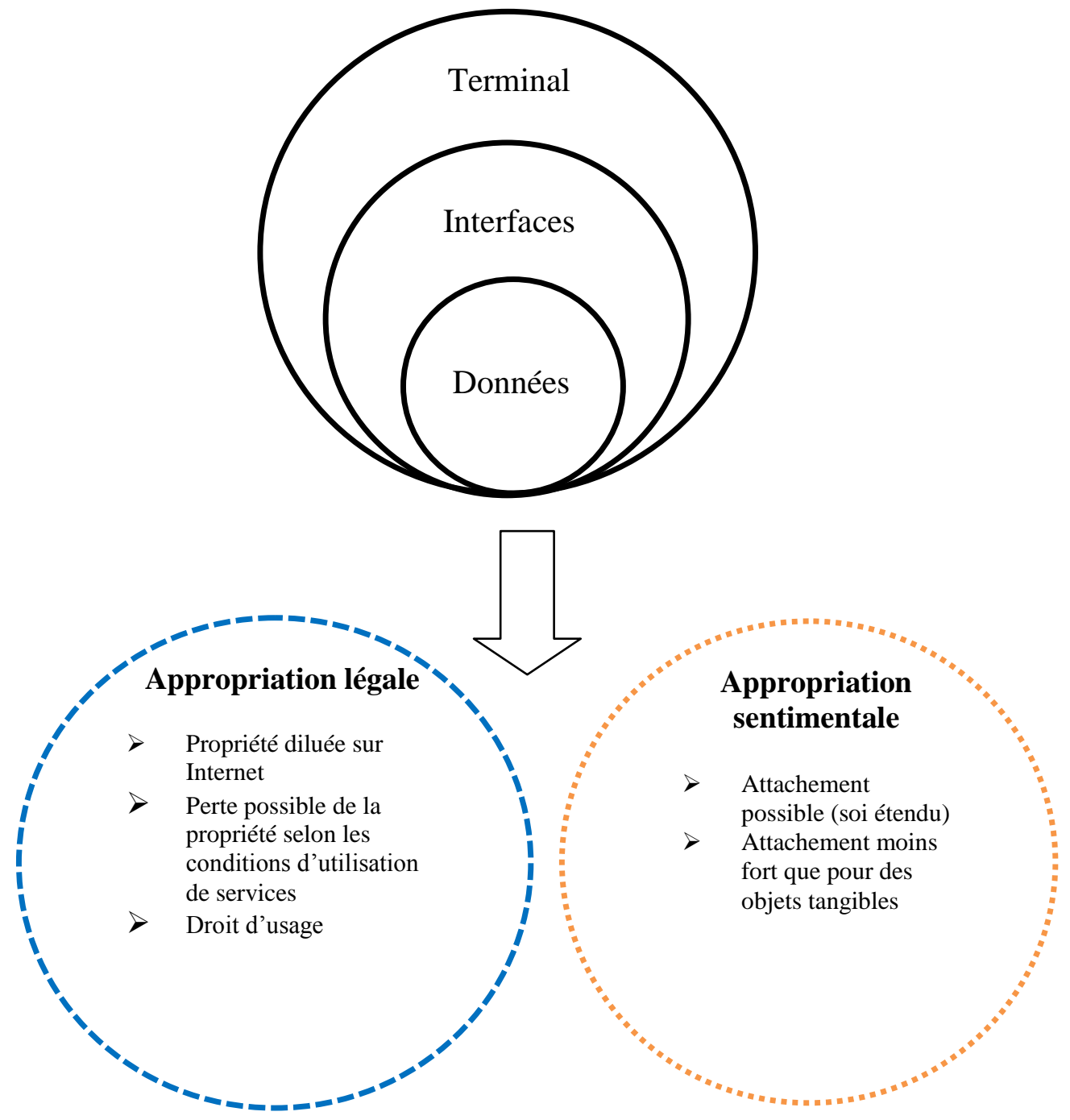

Cependant, à quoi correspondent les objets numériques pour les usagers? S'en sentent-ils propriétaires ? Y sont-ils attachés sentimentalement? Les réponses à ces questions ont émergé d'une série d'entretiens qualitatifs, elles sont présentées ci-après.

\section{APPROPRIATION DES OBJETS NUMERIQUES : LE POINT DE VUE DES UTILISATEURS}

Si la section précédente porte un regard théorique sur les objets numériques, cette section a pour objectif de confronter théorie et pratiques des utilisateurs. Pour ce faire, nous avons réalisé 
une étude qualitative (2-1), permettant de mieux comprendre les individus au sujet des objets numériques et de leur appropriation (2-2).

\section{2-1. Méthodologie de la recherche}

Dans une perspective exploratoire, nous avons voulu savoir comment les individus appréhendaient la notion d'objets numériques. Pour cela, 24 entretiens semi-directifs ont été réalisés à l'aide d'un guide d'entretien portant sur la relation au numérique (annexe 1). Afin d'identifier les différentes tactiques possibles en matière de gestion de ses objets numériques, le profil des répondants a été diversifié sur la base de leur âge (de 18 à 80 ans) et de leur degré d'expertise relatif aux technologies de l'information et de la communication (annexe 2). Le degré d'expertise de l'individu (« expert», «intermédiaire », «non expert ») a été déterminé au regard de la profession en lien ou non avec le numérique (ex : ingénieur en informatique) et d'une autoévaluation de son propre niveau. En effet, le critère subjectif est dans ce domaine aussi utile que des critères plus objectifs (Perraud, 2013). Les entretiens qui ont duré entre 21 et 88 minutes (moyenne : 40 minutes) - ont été intégralement retranscrits. Après lecture des entretiens, une grille d'analyse a été élaborée par les chercheurs, sur la base des thèmes apparaissant dans les divers entretiens (Huberman et Miles, 1991). Une analyse de contenu manuelle a été menée en deux temps. Dans un premier temps, des analyses verticales, entretien par entretien, ont été effectuées. Dans un second temps, une analyse horizontale a permis de déceler les similitudes et différences entre les entretiens.

\section{2-2. Résultats}

Nous restituons ici les résultats en trois volets : la perception qu'ont les utilisateurs des objets numériques, l'appropriation légale, puis sentimentale.

La perception des objets numériques : un statut ambigu. Au début de chaque entretien, il a été systématiquement demandé au répondant de nous décrire les objets numériques qu'il possédait. Les trois dimensions des objets numériques ressortent bien : les données numériques ellesmêmes, les interfaces les «traduisant » et les terminaux physiques permettant de les manipuler (tableau 1).

Tableau 1 : Qu'est-ce qu'un objet numérique pour les utilisateurs ?

\begin{tabular}{|c|c|c|}
\hline Des terminaux physiques & Des interfaces & Des données et fichiers \\
\hline
\end{tabular}




\begin{tabular}{|c|c|c|}
\hline $\begin{array}{l}\text { Ordinateur } \\
\text { Smartphone } \\
\text { Télévision } \\
\text { Camescope } \\
\text { Lecteur Mp3/Mp4 } \\
\text { Box internet } \\
\text { Clé USB } \\
\text { Disque dur } \\
\text { Console de jeux } \\
\text { Liseuse de livres électroniques } \\
\text { WebCam }\end{array}$ & $\begin{array}{l}\text { Logiciels de bureautique } \\
\text { (Word, Excel, Open office, } \\
\text { Adobe reader) } \\
\text { Logiciels de messagerie } \\
\text { Logiciels de jeux }\end{array}$ & $\begin{array}{l}\text { De la musique (MP3) } \\
\text { Des films } \\
\text { Des photos } \\
\text { Des images } \\
\text { Des vidéos } \\
\text { Des fichiers texte } \\
\text { Des documents scannés (de } \\
\text { type administratif) } \\
\text { Des e-book } \\
\text { Des e-mails }\end{array}$ \\
\hline
\end{tabular}

Dans le détail, notons que tous nos répondants citent en premier le terminal physique (ordinateur, tablette, smartphone, etc.). Seuls deux experts ont évoqué spontanément les données numériques : "Un objet numérique, ça peut être une entité qui est représentée par des 0 et des 1. Ça peut être pas mal de choses ! Ça peut être de l'audio, de la vidéo, du texte, des images évidemment puisque j'ai dit vidéo... Un peu tout, quoi!» (n¹6, H, 63 ans, E). Pour les autres, les données et les interfaces ne sont citées qu'après nos relances et demandes de précisions. Cela traduit une réelle imbrication - voire une absence de distinction - entre les données numériques immatérielles et les supports physiques permettant de les prendre en main. Pour nos répondants, une photo numérique n'est rien sans un ordinateur, une tablette, un cadre ou un smartphone permettant de la visualiser ou de la stocker/conserver. De même le fichier Mp3 prend tout son sens associé à un lecteur Mp3, un smartphone ou encore un ordinateur qui permet de l'écouter. Par exemple, $\mathrm{n}^{\circ} 2$ (F, 30 ans, NE) évoque d'une manière générale «Le Mp3 », sans faire la distinction entre les données numériques (le fichier Mp3) et le terminal physique (le baladeur $\mathrm{Mp} 3)$ : «le Mp3 est petit et on peut le prendre partout. ».

La propriété numérique liée au contrôle des objets numériques. Dans la grande majorité des cas, la question de la propriété n'est pas soulevée en situation « off-line ». Intuitivement, les répondants focalisent leur sentiment de propriété sur le terminal (mon ordinateur, ma tablette) ou sur les fichiers (mes mp3,...) mais jamais sur les interfaces.

A contrario, la question de la propriété se pose lorsque des services «online » sont utilisés pour stocker ou échanger des fichiers (cloud, réseaux sociaux, etc.). Certains répondants s'estiment être les propriétaires de fichiers musicaux numériques alors que ceux-ci ne leur appartiennent pas légalement car émanant du droit d'usage (la bibliothèque iTunes, par exemple). Cependant, la question de la propriété n'apparait qu'en filigrane avec la peur de perdre le contrôle des données : «des services qui pourraient proposer des services vraiment sécurisés pour ne pas se faire pirater nos données. Je pense que c'est vraiment important. Je pense qu'il y a beaucoup de gens qui ne stockent pas par internet à cause de ça. » (nº24, H, 18 ans, I). Malgré ces craintes, les répondants reconnaissent ne pas lire entièrement les modalités d'utilisation des sites internet. Les experts semblent néanmoins plus conscients des questions de propriété. En effet, dans notre échantillon, 6 des 9 experts disent se renseigner : "Je ne lis pas toutes les conditions d'utilisation. Il y a certains sites... certaines personnes sur internet qui, justement, se 
spécialisent entre guillemets, là-dedans, ressortent les points un petit peu importants des conditions d'utilisation. Et, quand je trouve que quelque chose est important, je vais me renseigner un peu. » $\left(\mathrm{N}^{\circ} 11, \mathrm{H}, 24\right.$ ans, E).

Finalement, la notion de confiance apparait ici déterminante. La confiance envers le prestataire est évoquée d'une part: «Je vérifie où sont stockées mes données... elles sont stockées sur le sol français, c'est-à-dire qu'il faut un engagement de la part de l'hébergeur [à respecter les lois françaises] ». $\left(\mathrm{n}^{\circ} 18, \mathrm{H}, 45\right.$ ans, E) et la confiance envers les autres utilisateurs d'autre part. Sur ce dernier point, le répondant $\mathrm{n}^{\circ} 13$ (H, 24 ans, E) résume la situation «Lorsqu'on s'inscrit sur ce genre de sites, il y a toujours une grande page avec des conditions d'utilisation qui doit bien prendre une bonne journée à lire et tout le monde clique sur la petite case en bas "j'ai compris et j'accepte les conditions ", sans regarder ce qu'il y a marqué. Donc, au final, je pense que les lois y sont, les conditions y sont aussi, mais personne ne prend le temps de les lire parce que c'est comme lire les petites lignes du contrat auquel on souscrit et qui font trois pages. Donc, on fait confiance à ce que nous disent les gens autour de nous qui ont déjà utilisé ça [...] Pourtant ça pose de nombreux problèmes. Des photos que les gens ont vues [sur Facebook], qu'ils ont voulu retirer et qu'ils n'ont pas pu parce que, au final, ce ne sont pas leurs photos, mais celles de Facebook».

L'attachement sentimental aux possessions numériques: résultante d'un processus d'appropriation $d u$ support. Sans surprise, les objets numériques, dans leurs trois dimensions imbriquées, apparaissent globalement associés à des pratiques quotidiennes, ancrées dans le présent à travers les notions d'accessibilité, d'instantanéité et de partage. Les liens affectifs n'apparaissent que suite à des tactiques d'appropriation qui diffèrent selon le but recherché : retrouver un sens du passé ou se projeter dans le futur. Pour préserver des objets numériques ayant trait à la mémoire et au passé de l'individu, la tendance semble être la tangibilisation d'objets numériques. Inversement, lorsque l'individu a l'intention de conserver dans le temps, des objets physiques auxquels il est attaché, notamment pour favoriser leur transmission, il effectue l'opération inverse. Nous observons ainsi une tendance à la dématérialisation d'objets physiques ; c'est alors l'objet physique, tangible, qui devient insuffisant.

\section{1) Une tendance à tangibiliser les objets numériques porteurs de souvenirs du passé}

Nos résultats indiquent que les individus peuvent développer une relation affective avec un objet numérique. Dans ce cas, la troisième dimension de l'objet numérique (le terminal) n'a aucune importance. Au contraire, les individus vont créer leur propre support physique pour se l'approprier. Ainsi, nos répondants ressentent le besoin de réifier l'objet virtuel : «Je préfère la photo imprimée. Ça rappelle plus le souvenir. Je ne sais pas, l'ordinateur, ça me bloque. Je ne ressens pas la même chose quand je l'ai dans la main qu'en regardant sur un écran. » $\left(\mathrm{n}^{\circ} 23, \mathrm{~F}, 57\right.$ ans, NE). L'objet physique apparaît plus facile à s'approprier, à engendrer une valeur sentimentale pour l'individu : "C'est sûr que format papier, c'est plus sentimental. Lorsqu'on les développe, c'est plus pour garder un vrai souvenir, alors que photo numérique, c'est pour stocker plein de photos, que ce soit sentimental ou non. » $\left(\mathrm{n}^{\circ} 24 ; \mathrm{H}, 18\right.$ ans, I). Cette tendance semble d'ailleurs indépendante de l'âge de l'individu : $\mathrm{n}^{\circ} 11(\mathrm{H}, 24$ ans, E) exprime le besoin d'avoir un support physique pour les objets numériques auxquels il est attaché. Par exemple, il garde les CD et les boîtes des jeux qu'il 
aime (dont Final Fantasy) et avec lesquels il joue depuis plus de 10 ans. "C'est des souvenirs, ça reste, [...] des jeux que je joue depuis 10-12 ans [...] C'est très très important».

En fonction de la nature de la possession numérique concernée, la tactique de tangibilisation prendra ainsi la forme d'une impression (documents, photos), d'une gravure (musiques, vidéos), ou de l'achat d'un support physique (CD de jeu vidéo, DVD des films préférés). Les raisons de tangibilisation qui apparaissent dans les propos des répondants sont alors les suivantes :

- pour pouvoir toucher les objets auxquels on attaché : "Pour moi c'est tellement virtuel, c'est pas palpable », ( $\mathrm{n}^{\circ} 19, \mathrm{H}, 64$ ans, E). L'individu ressent le besoin de toucher et de conserver les objets qui ont forgé son identité et qu'il considère comme faisant partie de son soi étendu.

- pour protéger les objets auxquels on tient : «Le numérique c'est éphémère, c'est pas sécurisé » $\left(\mathrm{n}^{\circ} 15, \mathrm{~F}, 23\right.$ ans, E).

- pour les exposer, que ce soit pour soi : «mes boîtes de jeu, elles sont exposées dans ma chambre, c'est des souvenirs, ça compte pour moi » $\left(\mathrm{n}^{\circ} 11, \mathrm{H}, 24 \mathrm{ans}, \mathrm{E}\right)$, ou pour les autres : «les photos qu'on aime, elles sont imprimées et mises dans un album [...] On va le chercher quand la famille est là » $\left(\mathrm{n}^{\circ} 19, \mathrm{H}, 64\right.$ ans, E).

Cependant, lorsqu'un objet est important pour un individu, celui-ci souhaite le conserver dans le futur, pour éventuellement le transmettre aux générations suivantes. Dans ce cas, quand l'objet chéri est initialement physique, la personne aura tendance à le convertir en objet numérique.

\section{2) Une tendance à dématérialiser les possessions tangibles ayant trait au futur}

Le numérique apparaît comme un moyen de construire et d'entretenir son identité personnelle, familiale ou collective, pour qu'elle perdure dans le futur. Pour ce faire, les tactiques utilisées sont la dématérialisation de possessions tangibles et la sélection des possessions numériques à sauvegarder. La détérioration des objets physiques (photos, vinyles, diapositives, correspondances) est citée comme la raison de la dématérialisation : " ce qu'on compte faire c'est numériser nos diapos, pour faire des albums du passé, parce que des diapos on en a beaucoup. [...] Parce que les diapos s'altèrent [...]. Les numériser, c'est aussi les sécuriser. On voit que ça se dégrade un peu. Pour les conserver $"\left(\mathrm{n}^{\circ} 19, \mathrm{H}\right.$, 64 ans, E).

L'idée sous-jacente à cette dématérialisation est la volonté de préserver un objet pour qu'il reste intact malgré le passage du temps et ainsi le rendre susceptible de faire partie d'un patrimoine familial que l'individu souhaite transmettre : "Avant, il fallait les négatifs et souvent, on perd les négatifs. Chose que, avec le numérique, tu peux refaire.» (n॰5, F, 58 ans, NE) ou encore " J'avais fait une photo de ma grand-mère que j'avais numérisé en fait et puis sous Photoshop j'avais recréé les pixels manquants; et puis des traces de pliures de la photo, donc comme on peut agrandir pas mal, le pixel apparait en carré, alors c'est assez grand quand on agrandit, donc on va chercher la couleur, et c'était bien. J'ai tiré la 
photo sur une imprimante photo en fait. Maintenant je vais le faire... Comme c'était mon arrière-grand-mère, donc celle-là je l'avais faite mais maintenant je compte en faire aussi ; d'autres photos de famille, comme mon frère $m$ 'en a envoyé, essayer de retravailler un peu ça. » ( $\mathrm{n}^{\circ} 19, \mathrm{H}, 64$ ans, E).

Cependant, seuls les documents jugés importants font l'objet de sauvegardes : "C'est certain que j'ai des disquettes qui datent de 1983, je ne suis pas sûr de pouvoir les lire à cette heure-ci. Mais je pense que ça n'a plus de valeur. [...] Moi je fais la sélection. On a fait cette évolution » $\left(\mathrm{n}^{\circ} 18, \mathrm{H}, 45\right.$ ans, E). Les problèmes de compatibilité entre les fichiers actuels que l'individu souhaite transmettre et les ordinateurs ou logiciels du futur sont évoqués, notamment par les experts : "J'espère que tout ce qui est ancien en informatique en stockage de données sera lisible dans le temps par certains moyens électroniques et informatiques » ( $\left.\mathrm{n}^{\circ} 19, \mathrm{H}, 64 \mathrm{ans}, \mathrm{E}\right)$; "Quelque part, je me dis que ce qu'on a aujourd'hui, c'est presque inutile de le conserver, puisque les systèmes d'exploitation de 20-30-40 ans n'existeront plus." (n॰13, H, 24 ans, E) ; «Un fichier pdf, on est certain de le lire 25 ans plus tard, alors qu'un fichier word de 10 ans, tu pourras peut-être pas le lire avec une future version $»\left(\mathrm{n}^{\circ} 18, \mathrm{H}, 45\right.$ ans, $\left.\mathrm{E}\right)$.

\section{DISCUSSION : TYPOLOGIE D'ATTITUDES A L'EGARD DES OBJETS NUMERIQUES}

La revue de littérature et l'étude qualitative présentées précédemment nous amènent à retenir les notions de propriété (appropriation légale) et de relation affective (appropriation sentimentale). Ces différents éléments vont conduire à un traitement différencié des objets numériques, selon le type de données, l'attachement affectif et le droit de propriété perçu. Trois configurations d'objets émergent et mettent ainsi en lumière des problématiques différentes (tableau 2).

Tableau 2 : Les différentes configurations d'objets numériques et problématiques soulevées

\begin{tabular}{|c|c|c|c|c|}
\hline Configuration & $\begin{array}{c}\text { Objets numériques } \\
\text { concernés : } \\
\text { dimensions et } \\
\text { exemples }\end{array}$ & $\begin{array}{c}\text { Caractéristiques } \\
\text { de } \\
\text { l'appropriation } \\
\text { sentimentale }\end{array}$ & $\begin{array}{c}\text { Caractéristiques } \\
\text { de } \\
\text { l'appropriation } \\
\text { légale }\end{array}$ & $\begin{array}{c}\text { Problématiques } \\
\text { soulevées }\end{array}$ \\
\hline $\begin{array}{l}\text { Les objets } \\
\text { numériques } \\
\text { fonctionnels }\end{array}$ & $\begin{array}{l}\text { Terminal : smartphone, } \\
\text { ordinateur, etc. avec son } \\
\text { contenu }\end{array}$ & Faible & $\begin{array}{l}\text { Sentiment de } \\
\text { propriété juridique } \\
\text { fort } \\
\text { Mais qui ne } \\
\text { correspond pas } \\
\text { forcément à la réalité }\end{array}$ & $\begin{array}{l}\text { La confiance dans les } \\
\text { usages numériques. }\end{array}$ \\
\hline
\end{tabular}




\begin{tabular}{|c|l|l|l|l|}
\hline $\begin{array}{c}\text { Les objets } \\
\text { numériques } \\
\text { miroirs }\end{array}$ & $\begin{array}{l}\text { Interface : comptes mail, } \\
\text { réseaux sociaux alimentés } \\
\text { de données, bibliothèque } \\
\text { iTunes, blogs. }\end{array}$ & $\begin{array}{l}\text { Progressive : } \\
\text { accumulation passive } \\
\text { de données / } \\
\text { d'interfaces online } \\
\text { connuisant à une } \\
\text { appropriation } \\
\text { sentimentale au fil du } \\
\text { temps }\end{array}$ & $\begin{array}{l}\text { Sentiment de } \\
\text { propriété juridique } \\
\text { variable mais qui ne } \\
\text { correspond pas } \\
\text { forcément à la réalité }\end{array}$ & $\begin{array}{l}\text { Mémoire et temps } \\
\text { numérique }\end{array}$ \\
\hline $\begin{array}{c}\text { Les objets } \\
\text { numériques } \\
\text { totems }\end{array}$ & $\begin{array}{l}\text { Données importantes aux } \\
\text { yeux de l'utilisateur qui } \\
\text { sont associées à une } \\
\text { contrepartie physique }\end{array}$ & $\begin{array}{l}\text { Forte : action } \\
\text { conduisant à une } \\
\text { appropriation } \\
\text { sentimentale } \\
\text { tangibilisation ou } \\
\text { dématérialisation). }\end{array}$ & $\begin{array}{l}\text { Sentiment de } \\
\text { propriété juridique } \\
\text { fort } \\
\text { Correspond à la } \\
\text { réalité }\end{array}$ & $\begin{array}{l}\text { Identité et } \\
\text { transmission } \\
\text { intergénérationnelle }\end{array}$ \\
\hline
\end{tabular}

\section{3-1. Les objets numériques fonctionnels}

Les objets numériques fonctionnels sont des objets qui facilitent la vie et les échanges quotidiens. Pour nos répondants, les trois dimensions de l'objet numérique ne sont pas dissociées dans cette configuration. Il peut donc s'agir d'un terminal (avec ses interfaces et données), d'une interface (avec ses données) ou uniquement de données. Par exemple, pour un individu, un objet numérique fonctionnel peut être son smartphone qui contient l'ensemble de ses photos numériques stockées dans le cloud; ou encore son ordinateur avec ses fichiers de travail, son compte mail avec l'ensemble de sa correspondance, etc. Comme leur nom l'indique, ces objets sont valorisés pour leur fonction pratique. Par conséquent, l'attachement sentimental est peu développé. Cela ne veut pas dire que ce sentiment ne viendra pas plus tard. Par exemple, si un individu s'attache à son smartphone qui contient «toute sa vie», l'objet va changer de configuration et devenir un objet « miroir », voire « totem ».

Puisqu'il y a imbrication des dimensions, le sentiment de propriété portant initialement sur le terminal peut se reporter sur les données qu'il contient. Dans ce cas, cette perception n'est pas toujours justifiée au regard du droit, notamment lorsque ces données sont stockées chez un prestataire extérieur (les données sur une interface en ligne telles que des documents stockés en cloud). Les préoccupations des usagers se reportent alors sur la notion de contrôle des données. Dès lors, la notion de confiance est déterminante. Elle s'incarne à deux niveaux : la confiance organisationnelle envers les prestataires de service, notamment pour les experts, et la confiance interpersonnelle envers les pairs -les autres utilisateurs de la communauté numérique- qui signaleraient les éventuels problèmes. En ce sens, notre recherche permet de contribuer au courant de recherche qui souligne l'importance de la confiance dans les usages numériques (Lobet-Maris et al., 2009).

\section{3-2. Les objets numériques miroirs}

Dans la lignée de la configuration précédente, l'individu accumule dans le temps de multiples données numériques (généralement associées à des interfaces - online ou offline). Par exemple, il peut s'agir d'une accumulation de photos numériques, de pages de son blog personnel ou encore d'informations sur ses comptes de réseaux sociaux, de fichiers dans sa bibliothèque numérique, etc. Cette masse d'informations devient peu à peu le reflet d'une partie de soi (d'où la 
terminologie employée). Par exemple, les e-mails mis bout à bout permettent de retracer la genèse et de représenter la relation existant entre deux personnes. A noter que pour les objets online, cette présentation de soi est en partie standardisée à travers les template des interfaces comme les sites de réseaux sociaux, ou des blogs (Schau et Gilly, 2012). En raisonnant au niveau de la donnée, on peut alors parler de collection d'objets. Cependant, la collection de données dans son ensemble peut être considérée comme un objet à part entière : tel est le cas du compte Facebook ou encore des produits Apple (Belk, 2013). Nos résultats montrent que l'attachement sentimental peut être lié à la quantité de données, à l'investissement en argent (la bibliothèque iTunes par exemple) ou en temps (le nombre d'heures passées à jouer à un jeu en ligne, le temps passé sur le compte Facebook). Cette appropriation se fait petit à petit et de manière plus ou moins inconsciente, ce qui peut expliquer pourquoi les personnes rencontrées en aient peu parlé.

Ces constats rejoignent les réflexions sociologique et anthropologique sur la redéfinition du concept de mémoire. Au regard des sociologues et historiens des siècles prochains, nous serons probablement la génération la plus sincère de l'histoire tant il nous est difficile de contrôler les multiples traces numériques de nous-mêmes laissées sur le net (Sumit, 2012). La gestion de tous nos objets numériques s'avère parfois complexe et les individus se laissent bien souvent déborder par l'ampleur de la tâche. Par exemple, les individus sont parfois désemparés devant le fait de devoir trier des milliers de photos numériques, si bien qu'ils finissent par abandonner (Jonas, 2007). Une difficulté supplémentaire réside dans le fait que comme dans la configuration précédente, la propriété juridique n'est pas forcément établie, notamment si les données sont liées à des interfaces régies par le droit d'usage. Cela peut poser des questions dans l'avenir si l'individu souhaite en faire des objets totems.

\section{3-3. Les objets numériques «totems »}

La dernière configuration concerne celle des objets numériques sacralisés ou totems. Celle-ci peut être un prolongement de la configuration précédente, lorsque l'individu va agir sur les objets numériques. Cette fois-ci, l'appropriation sentimentale n'est plus passive (par accumulation inconsciente) mais active (par sélection ou création des objets).

En ce qui concerne les objets numériques off-line, la pratique est double. L'individu va soit s'approprier le support en réifiant la donnée virtuelle; soit créer une donnée virtuelle en dématérialisant un objet tangible auquel il tient - le plus souvent une photographie. Notons que le problème de la propriété juridique ne se pose pas dans cette configuration puisque l'individu sort l'objet du monde numérique ou qu'il possède son équivalent tangible.

Si la littérature suggérait un attachement plus difficile aux possessions numériques, cette étude révèle davantage une relation d'interdépendance entre les possessions tangibles auxquelles l'individu est attaché et leurs équivalents dématérialisés. En outre, nous observons, dans cette configuration, des différences générationnelles contraires à celles évoquées dans la littérature antérieure. Cette dernière suggérait un attachement aux objets numériques plus développé chez les moins de 60 ans (Cushing, 2012). Or, dans notre recherche, il semble que les plus âgés ont davantage tendance à dématérialiser leurs possessions tangibles pour les conserver en version numérique, ou tout du moins à s'exprimer davantage que les plus jeunes à ce sujet. C'est peutêtre parce qu'ils sont les plus aptes à endosser le rôle social de "passeurs de mémoire ». D'ailleurs, des services pour favoriser ces tactiques de tangibilisation ou de dématérialisation se développent à l'initiative d'entreprises privées ou d'organismes publics. A titre d'exemple, des 
services proposent de créer des livres-albums de photos; favorisant ainsi des actions du numérique vers le tangible. Inversement, des institutions publiques proposent de recueillir des photos argentiques et de les convertir en numérique pour des archives publiques et leur rôle de mémoire. C'est ainsi que l'opération Grande Collecte, portée entre autres par la Bibliothèque Nationale de France, invitait les particuliers à venir déposer les archives qu'ils conservaient sur la période 1914-1918 afin de les numériser et les déposer sur la base de données européenne des souvenirs de la Grande Guerre ${ }^{3}$.

En ce qui concerne les pratiques on-line, la réappropriation des objets numériques miroirs n'a pas été directement observée dans notre échantillon. Pourtant, à partir du moment où les individus prennent conscience de l'accumulation de documents biographiques tangibles, ils cherchent à les trier, à les organiser, à les expliquer, à leur donner du sens, et donc à devenir historiens de leurs propres souvenirs (Guillemot, 2010). Cette différence laisse supposer que l'usage du numérique est trop récent et que ces comportements devraient apparaître plus tard. C'est en tout cas le pari de plusieurs prestataires qui développent des services pour aider les individus à créer leur propre «muséographie personnelle »: Memory-life ou 109lab proposent par exemple d'organiser et de mettre en scène nos traces en ligne, tandis que 1000memories assiste le transfert des photos argentiques vers les réseaux sociaux (Merzeau, 2013). D'autres prestataires de services (ex: passwordbox), vont plus loin et proposent de conserver les identifiants de vos divers comptes en ligne pour pouvoir les transmettre aux générations futures (héritage numérique).

\section{CONCLUSION}

L'objectif de cette recherche était de porter un regard sur les objets numériques et plus particulièrement sur la manière dont les individus se les approprient. Nos principaux résultats sont les suivants :

- Nous observons une imbrication des objets numériques, avec des dimensions tangibles (terminal) et intangibles (interfaces et données) difficilement dissociables.

- $\quad$ Il en résulte que la question de la propriété juridique ne se pose pas vraiment, à moins d'être confronté à la perte de contrôle des données numériques.

- Nous mettons à jour des tactiques d'appropriation affectives par tangibilisation et dématérialisation des objets numériques par les individus.

- Il en découle trois configurations d'objets numériques à travers desquelles le prisme temporel apparait déterminant : objet fonctionnel (présent), objet miroir (passé) et objet totem (projection future).

Il s'agit désormais d'engager une réflexion sur l'adaptation des règles juridiques appliquées aux questions de l'identité et des possessions sur internet (Iteanu, 2008), particulièrement celles liées aux temps (Crouzet, 2012) et de réfléchir à une meilleure sensibilisation et information des usagers (Caroll et Romano, 2010). Dans tous les cas, les constats soulevés dans cette recherche

\footnotetext{
${ }^{3} \mathrm{http}: / /$ centenaire.org/fr/tresors-darchives/fonds-prives/archives/retour-sur-la-grande-collecte-une-selectiondarchives
} 
nécessiteraient d'être approfondis dans une perspective interdisciplinaire avec des considérations, entre autres, anthropologiques, sociologiques, juridiques, ou encore émanant des sciences de gestion (particulièrement en comportement des consommateurs et en management des systèmes d'information).

\section{BIBLIOGRAPHIE}

Bardhi F. et Eckhardt G.M (2002), Access-Based Consumption: The Case of Car Sharing, Journal of Consumer Research, 39, 4, 881-98

Bellamy G., Arnold M., Gibbs M., Nansen B. et Kohn T. (2014), Consumer issues for planning and managing digital legacies, IEEE Technology and Society, Magazine, fall, 26-31.

Belk R. (1988), Possessions and the Extended Self, Journal of Consumer Research, 15, 139-168

Belk R. (2013), Extended Self in a Digital World, Journal of Consumer Research, 40, 3, 477-500

Carroll E. et Romano J. (2010), Your Digital Afterlife: When Facebook, Flickr and Twitter Are Your Estate, What's Your Legacy? Berkeley, CA: New Rider's Press

Coutant A. et Stenger T. (2013), Identités Numériques, Paris : L'Harmattan

Crouzet J. (2012), Mourir en ligne: les héritiers peuvent-ils accéder aux données du défunt?, Mémoire de Master in Science in Law \& Tax management, EDHEC Business School sous la direction de Cédric Manara

Cushing A.L. (2012), Possessions and Self Extension in Digital Environments: Implications for Maintaining Personal Information, PhD dissertation, School of Information and Library Science, University of North Carolina at Chapel Hill

Denegri-Knott J., Watkins R. et Wood J. (2012), Transforming Digital Virtual Goods into Meaningful Possessions, in Molesworth M. et Denegri-Knott J. (Eds), Digital Virtual Consumption, London: Routledge, 76-91

Guillemot S. (2010), Les motivations des personnes âgées au récit de vie et leurs influences sur la consommation de services biographique. Thèse de doctorat en Sciences de Gestion. IAE Brest

Huberman A. M. et Miles M. B. (1991), Analyse des données qualitatives - Recueil de nouvelles méthodes. Bruxelles : De Boeck Université

Iteanu O. (2008), L'identité numérique en question, Paris : Eyrolles

Jonas I. (2007) La photographie de famille au temps du numérique, Enfances, Familles, Générations, 7, 93-111

Kirk D.S. et Sellen A. (2010), On Human Remains: Values and Practice in the Home Archiving of Cherished Objects, ACM Transactions on Computer-Human Interaction, 17, 3, 10

Kleine S.S, Kleine R.E. et Allen C.T. (1995), How is a Possession « Me » or « Not Me »? Characterizing types and an antecedent of material possession attachment, Journal of Consumer Research, 22, 327-343

Llamas R. et Belk R. (2012), Living in a digital world, in Belk R. and Llamas R. (Eds), The Routledge Companion to Digital Consumption, New York: Routledge, 3-12

Lobet-Maris, C., Lucas, R., \& Six, B. (2009), Variations sur la confiance: Concepts et enjeux au sein des théories de la gouvernance, Bruxelles: P.I.E. Peter Lang

Magaudda P. (2012), What happens to materiality in Digital Virtual Consumption? In Molesworth M. et Denegri-Knott J. (Eds), Digital Virtual Consumption, London: Routledge, $111-126$ 
Merzeau L. (2013), Identity commons : du marquage au partage, in Coutant A. et Stenger T. (Ed), Identités numériques, Paris : L'Harmattan, 35-49

Perraud L. (2013), Antécédents et conséquences de l'intrusion publicitaire perçue sur internet: le cas des formats publicitaires, Thèse de doctorat en sciences de gestion, Université de Bourgogne

Schau H.J and Gilly M.C. (2012), From freeform to templates. The evolution of self-presentation in Cyberia, in Belk R. and Llamas R. (Eds), The Routledge Companion to Digital Consumption, New York: Routledge, 72-79

Sears N. (2012), Bruce Willis fights to leave his iPod tunes to his family: Actor considering legal action against Apple in battle over who owns songs downloaded from iTunes, DailyMail online edition, 4th September 2012

Siddiqui S. et Turley D. (2006), Extended the Self in a digital world, Advances in Consumer Research, Vol.33, ed. Pechmann C. and Price L., Duluth, MN: Association for Consumer Research, 647-648.

Sumit P-C (2012), Digital legacy : The fate of your online soul, New Scientist, Issue 2809, 40-43

Venkatesh A. et Dunkle D (2012), Digitizing physical objects in the home, in Belk R. and Llamas R. (Eds), The Routledge Companion to Digital Consumption, New York: Routledge, $13-27$ 
Annexe 1. Trame du guide d'entretien

Thème 1. Les objets numériques

$>$ Si je vous dis objet numérique, à quoi pensez-vous ?

$>$ Que possédez-vous en numérique ? + relances (demandes de précisions)

$>$ Quelles valeurs attribuez-vous à ces objets? + relances visant à déterminer l'attachement à ces objets

Thème 2. L'usage et la préservation des objets numériques

$>$ Comment utilisez-vous ces objets + relances (utilisation, cloud, sauvegarde, stockage, etc.)

$>$ La préservation de ces objets vous préoccupe-t-elle ?

$>$ Comment faites-vous pour préserver ces objets?

$>$ Que savez-vous des textes législatifs/ conditions légales d'utilisation des services que vous utilisez?

Thème 3. La transmission de ces objets numériques

$>$ Avez-vous déjà pensé à ce qui pourrait advenir de ces contenus dans le futur ?

$>$ Avez-vous déjà eu envie de transmettre vos contenus numériques ? (laisser l'ambigüité sur le terme transmettre $=$ donner, cadeau, etc.)

Différences avec la transmission de leur contrepartie classique ?

$>$ Avez-vous des besoins et des attentes de produits/services pouvant vous aider dans cette démarche?

Dans la dernière partie de l'interview, nous avons recueilli l'avis des personnes sur des services de Legs Digital (passwordbox, Edeneo, etc.)-Ce point n'est pas traité dans cet article.

Merci de votre participation, voyez-vous quelque chose à ajouter ? 
Annexe 2 : Le profil des répondants

\begin{tabular}{|c|c|c|c|c|c|c|}
\hline Individu & Age & $\begin{array}{c}\text { Durée } \\
\text { entretien (mn) }\end{array}$ & $\begin{array}{c}\text { Niveau } \\
\text { d'expertise }\end{array}$ & Profession & Sexe & $\begin{array}{l}\text { Situation } \\
\text { familiale }\end{array}$ \\
\hline 1 & 32 & 21 & Non Expert (NE) & Commercial & $\mathrm{H}$ & En couple \\
\hline 2 & 30 & 36 & Non Expert (NE) & En congé parental & $\mathrm{F}$ & En couple \\
\hline 3 & 57 & 27 & Non Expert (NE) & $\begin{array}{c}\text { Manager Grande } \\
\text { distribution }\end{array}$ & $\mathrm{H}$ & Divorcé(e) \\
\hline 4 & 30 & 24 & Non Expert (NE) & Serveuse & $\mathrm{F}$ & En couple \\
\hline 5 & 58 & 48 & Non Expert (NE) & Secrétaire & $\mathrm{F}$ & Marié(e) \\
\hline 6 & 74 & 39 & Non Expert (NE) & Retraité, ex médecin & $\mathrm{H}$ & Marié(e) \\
\hline 7 & 80 & 59 & Non Expert (NE) & $\begin{array}{c}\text { Retraité, ex directeur } \\
\text { d'hôtel }\end{array}$ & $\mathrm{H}$ & Veuf(ve) \\
\hline 8 & 67 & 24 & Non Expert (NE) & $\begin{array}{c}\text { Retraité, ex clerc de } \\
\text { notaire }\end{array}$ & $\mathrm{F}$ & Veuf(ve) \\
\hline 9 & 60 & 44 & Non Expert (NE) & $\begin{array}{c}\text { Retraité, ex auxiliaire de } \\
\text { vie }\end{array}$ & $\mathrm{F}$ & Veuf(ve) \\
\hline 10 & 24 & 36 & Intermédiaire (I) & $\begin{array}{c}\text { Etudiant (M2 agro- } \\
\text { alimentaire) }\end{array}$ & $\mathrm{H}$ & Célibataire \\
\hline 11 & 24 & 48 & Expert (E) & $\begin{array}{l}\text { Elève ingénieur en } \\
\text { informatique }\end{array}$ & $\mathrm{H}$ & En couple \\
\hline 12 & 44 & 88 & Expert (E) & $\begin{array}{l}\text { Ingénieur de recherche } \\
(\mathrm{PhD})\end{array}$ & $\mathrm{H}$ & Divorcé(e) \\
\hline 13 & 24 & 40 & Expert (E) & $\begin{array}{l}\text { Elève ingénieur en } \\
\text { informatique }\end{array}$ & $\mathrm{H}$ & Célibataire \\
\hline 14 & 24 & 29 & Expert (E) & $\begin{array}{l}\text { Elève ingénieur en } \\
\text { informatique }\end{array}$ & $\mathrm{H}$ & Célibataire \\
\hline 15 & 23 & 47 & Expert (E) & $\begin{array}{c}\text { Elève ingénieur en } \\
\text { informatique }\end{array}$ & $\mathrm{F}$ & En couple \\
\hline 16 & 63 & 31 & Expert (E) & $\begin{array}{l}\text { Retraité, ex ingénieur de } \\
\text { recherche }\end{array}$ & $\mathrm{H}$ & En couple \\
\hline 17 & 70 & 25 & Expert (E) & $\begin{array}{l}\text { Retraité, ex ingénieur de } \\
\text { recherche }\end{array}$ & $\mathrm{H}$ & En couple \\
\hline 18 & 45 & 61 & Expert (E) & $\begin{array}{l}\text { Ingénieur en } \\
\text { informatique }\end{array}$ & $\mathrm{H}$ & Marié(e) \\
\hline 19 & 64 & 47 & Expert (E) & $\begin{array}{l}\text { Retraité, ex ingénieur } \\
\text { informatique }\end{array}$ & $\mathrm{H}$ & Marié(e) \\
\hline 20 & 21 & 36 & Intermédiaire (I) & $\begin{array}{c}\text { Etudiant (M2 agro- } \\
\text { alimentaire) }\end{array}$ & $\mathrm{H}$ & Célibataire \\
\hline 21 & 22 & 39 & Non Expert (NE) & $\begin{array}{c}\text { Etudiant (M2 agro- } \\
\text { alimentaire) }\end{array}$ & $\mathrm{F}$ & En couple \\
\hline 22 & 26 & 44 & Intermédiaire (I) & Technicien méthodes & $\mathrm{H}$ & En couple \\
\hline 23 & 57 & 41 & Non Expert (NE) & Sans profession & $\mathrm{F}$ & Célibataire \\
\hline 24 & 18 & 41 & Intermédiaire (I) & $\begin{array}{l}\text { Etudiant (L1 } \\
\text { informatique) }\end{array}$ & $\mathrm{H}$ & Célibataire \\
\hline
\end{tabular}

